\title{
RBPJ and MAML3: Potential Therapeutic Targets for Small Cell Lung Cancer
}

\author{
HIDEYA ONISHI ${ }^{1}$, SHU ICHIMIYA ${ }^{1}$, KOSUKE YANAI $^{1}$, MASAYO UMEBAYASHI $^{2}$, \\ KATSUYA NAKAMURA ${ }^{1}$, AKIO YAMASAKI ${ }^{1}$, AKIRA IMAIZUMI ${ }^{3}$, SHUNTARO NAGAI $^{4}$, \\ MUTSUNORI MURAHASHI ${ }^{5}$, HISANOBU OGATA ${ }^{5}$ and TAKASHI MORISAKI ${ }^{2}$ \\ Departments of ${ }^{1}$ Cancer Therapy and Research,${ }^{4}$ Surgery and Oncology, \\ Graduate School of Medical Sciences, Kyushu University, Fukuoka, Japan; \\ ${ }^{2}$ Fukuoka General Cancer Clinic, Fukuoka, Japan; \\ ${ }^{3}$ Shukoukai Inc., Tokyo, Japan; \\ ${ }^{5}$ Department of Advanced Cell and Molecular Therapy, Kyushu University Hospital, Fukuoka, Japan
}

\begin{abstract}
Background/Aim: Small cell lung cancer (SCLC) is still a deadly type of cancer for which there are few effective therapeutic strategies. Development of a new molecule targeting agent is urgently desired. Previously we showed that recombination signal binding protein for immunoglobulin-kappa-J region (RBPJ) and mastermindlike 3 (MAML3) are new therapeutic targets for pancreatic cancer. In the present study, we analyzed whether RBPJ/MAML3 inhibition could also be a new therapeutic strategy for SCLC. Materials and Methods: Using silencing of RBPJ/MAML3, proliferation, invasion, migration and chemosensitivity of SBC-5 cells were investigated. Results: RBPJ/MAML3 inhibition reduced Smoothened and HES1 expression, suggesting that RBPJ/MAML3 signaling was through Hedgehog and NOTCH pathways. In the analysis of cell functions, RBPJ/MAML3 inhibition significantly reduced proliferation and invasiveness via reduction of expression of matrix metalloproteinases. On the other hand, RBPJ/MAML3 inhibition also reduced chemosensitivity to cis-diamminedichlo-roplatinum and gemcitabine. Conclusion: These results suggest that RBPJ and MAML3 could be new therapeutic targets for SCLC, however, chemosensitivity may be reduced in combinational use with other chemo-therapeutic agents.
\end{abstract}

Correspondence to: Hideya Onishi, Department of Cancer Therapy and Research, Graduate School of Medical Sciences, Kyushu University, 3-1-1 Maidashi, Higashi-ku, Fukuoka 812-8582, Japan. Tel: +81926426220, Fax: +81926426221, e-mail: ohnishi@ surg1 .med.kyushu-u.ac.jp

Key Words: RBPJ, MAML3, SCLC, therapeutic target.
Small cell lung cancer (SCLC) remains one of the deadliest types of cancer. One reason for this may be that there are still few effective chemotherapy agents for the treatment of refractory SCLC. After chemotherapy and chemoradiotherapy, disease relapse is observed within approximately 515 months $(1,2)$. The development of novel therapeutic strategies is urgently required.

Both Hedgehog and NOTCH signaling pathways are related to morphogenesis. They are re-activated in several types of cancer and contribute to the regulation of some biological functions of cancer cells $(3,4)$. Recently, it was shown that Hedgehog signaling is a crucial requirement in SCLC and that it could be a therapeutic target (5-7). On the other hand, regarding NOTCH signaling, only NOTCH1 is well reported and NOTCH1 expression is associated with favorable prognosis in SCLC (8-10).

Recombination signal binding protein for immunoglobulinkappa-J region (RBPJ) and mastermind-like (MAML) are activators of NOTCH signaling as transcriptional factor and transcriptional co-activator, respectively $(11,12)$. We revealed that RBPJ and MAML3 are mediators of Smoothened (SMO) transcription, which is a driver gene of Hedgehog signaling, and that they contribute to the induction of malignant phenotype of pancreatic cancer (13).

In the present study, whether RBPJ and MAML3 might be therapeutic targets for SCLC, which is a type of refractory neuroendocrine cancer, was evaluated.

\section{Materials and Methods}

Cell culture and reagents. A human SCLC cell line (SBC-5: American Type Culture Collection, Manassas, VA, USA) was maintained in RPMI-1640 medium (Nacalai Tesque, Kyoto, Japan) supplemented with $10 \%$ fetal calf serum (FCS; Life Technologies Grand Island, NY, USA) and antibiotics (100 units/ml of penicillin 
and $100 \mu \mathrm{g} / \mathrm{ml}$ of streptomycin). Cell numbers were counted under light microscopy. Gemcitabine and cis-diamminedichloroplatinum (CDDP) were purchased from Eli Lilly (Indianapolis, IN, USA) and Bristol Myers Squibb (NY, NY, USA), respectively.

RNA interference. Small interfering RNA (siRNA) for MAML3 (ON-TARGET plus SMART pool, L-013813), siRNA for $R B P J$ (ON-TARGET plus SMART pool, L-007772) and negative control siRNA (ON-TARGET plus si CONTROL non-targeting pool, D001810) were purchased from Dharmacon RNA Technologies (Chicago, IL, USA). Cells $\left(2 \times 10^{5}\right.$ cells/well) seeded in 6-well plates were transfected with $100 \mathrm{nM}$ siRNA using Lipofectamine RNAiMAX Reagent (Thermo Fisher Scientific, Waltham, MA, USA) according to the manufacturer's instructions. Cells were assayed at 2 days after transfection.

Wound-healing assay. Cells transfected with control siRNA, RBPJ SiRNA or MAML3 siRNA were seeded onto a 24 -well plate at a density of $5.0 \times 10^{5}$ cells/well. After cells were confluent, a scratch was made using a $24-\mathrm{G}$ needle (Terumo, Tokyo, Japan). Images were captured at a magnification of $\times 100$ at intervals for 4 days.

Matrigel invasion assay and migration assay. The invasiveness of pancreatic cancer cells was assessed based on the invasion of cells transfected with control siRNA, RBPJ siRNA or MAML3 siRNA through Matrigel-coated transwell inserts as described previously (14). In brief, the upper surface of a filter (pore size, $8.0 \mu \mathrm{m}$; BD Biosciences, Heidelberg, Germany) was coated with basement membrane Matrigel (BD Biosciences). Cells were suspended in RPMI-1640 with $10 \%$ FCS then $8 \times 10^{4}$ cells were added to the upper chamber and incubated for $16 \mathrm{~h}$. After incubation, the filter was fixed and stained with Diff-Quick reagent (International Reagents, Kobe, Japan). All cells that had migrated from the upper to the lower side of the filter were counted under a light microscope (BX50; Olympus, Tokyo, Japan) at a magnification of $\times 100$. Tumor cell invasiveness testing was carried out in triplicate wells. Cell migration assay was performed in the same way using Matrigelnon-coated transwell inserts.

Western blotting. Cells transfected with control siRNA, RBPJ siRNA or MAML3 siRNA were treated with 10,100 , and 1000 $\mu \mathrm{g} / \mathrm{ml}$ of gemcitabine or 50, 500, and $1000 \mu \mathrm{g} / \mathrm{ml}$ CDDP for 2 days. Extraction of whole cells was then performed with M-PER Reagents (Pierce Biotechnology, Rockford, IL, USA) according to the manufacturer's instructions. Protein samples $(50 \mu \mathrm{g})$ were separated by electrophoresis on a sodium dodecyl sulfate-polyacrylamide gel and transferred to Protran nitrocellulose membranes (Whatman $\mathrm{GmbH}$, Dassel, Germany). The protein-transferred membranes were incubated overnight at $4^{\circ} \mathrm{C}$ with primary antibodies for matrix metalloproteinase (MMP) 2 (1:200, sc-10736), MMP9 (1:200, sc$6840)$, E-cadherin $(1: 200$, sc-7870), vimentin $(1: 200$, sc-6260y), SNAI1 (1:200, sc-10433), MAML3 (1:100, sc-82220), RBPJ (1:100, sc-8213), Smoothened (SMO) (1:200, sc-13943) and HES1 (1:100, sc-13844) from Santa Cruz Biotechnology, Dallas, TX, USA. Peroxidase-linked secondary antibodies (Amersham Biosciences, Piscataway, NJ, USA) were subsequently added and the membranes were further incubated for $1 \mathrm{~h}$ at room temperature. Antibody for $\alpha$-tubulin (1:1000; Sigma-Aldich, St. Louis, MO, USA) was used as protein loading control. Band intensities were analyzed qualitatively.

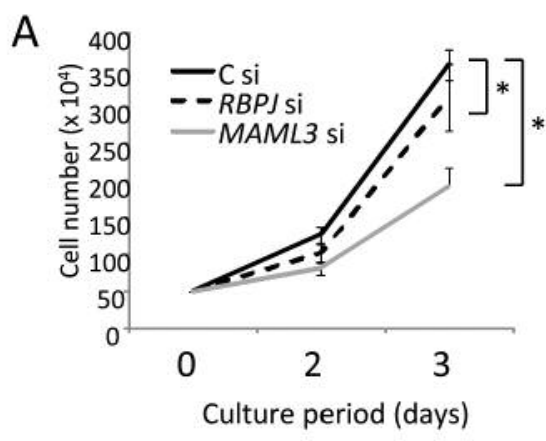

B
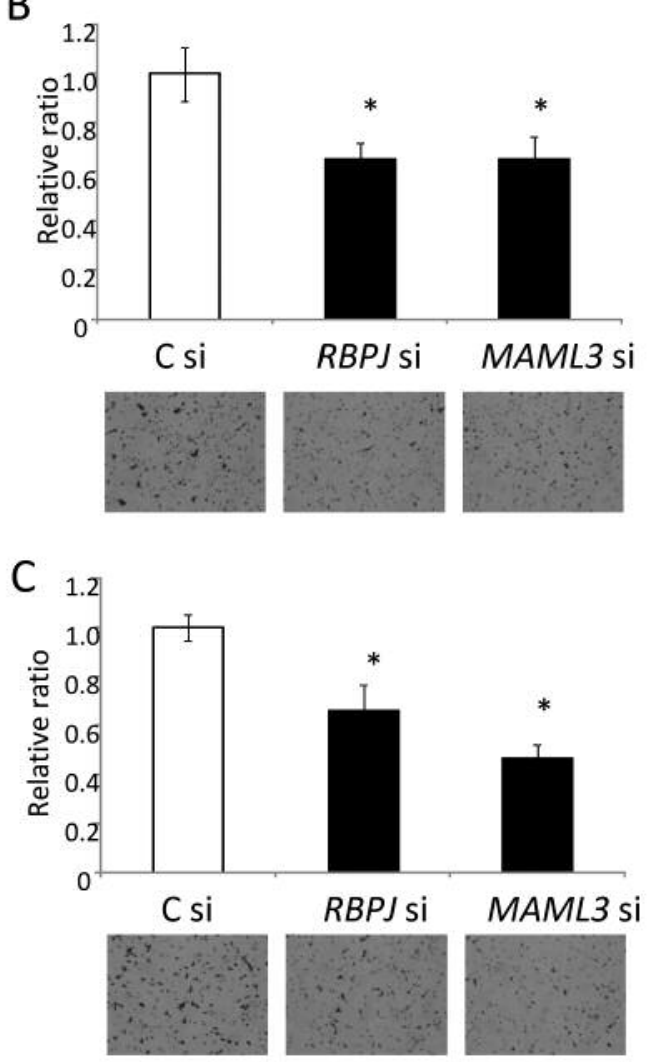

Figure 1. Inhibition of recombination signal binding protein for immunoglobulin-kappa-J region (RBPJ) and mastermind-like 3 (MAML3) reduced proliferation, migration and invasion in small cell lung cancer cells. RBPJ siRNA (si)-transfected, MAML3 si-transfected and control si (C si)-transfected SBC-5 cells were used in the experiments. A: Cell numbers were counted at the indicated days of culture using light microscopy. B: The number of cells which had migrated through nonmatrigel-coated membrane was counted. $C$ : The number of cells which had invaded through matrigel-coated membrane was counted. Representative images are shown $(\times 100)$. Data are means \pm standard deviation. *Significantly different from control at $p<0.05$.

Statistical analysis. The data are presented as means \pm standard deviation (SD). Student's $t$-tests were used to compare continuous variables between two groups. Differences with $p$-values of less than 0.05 were considered as statistically significant. 


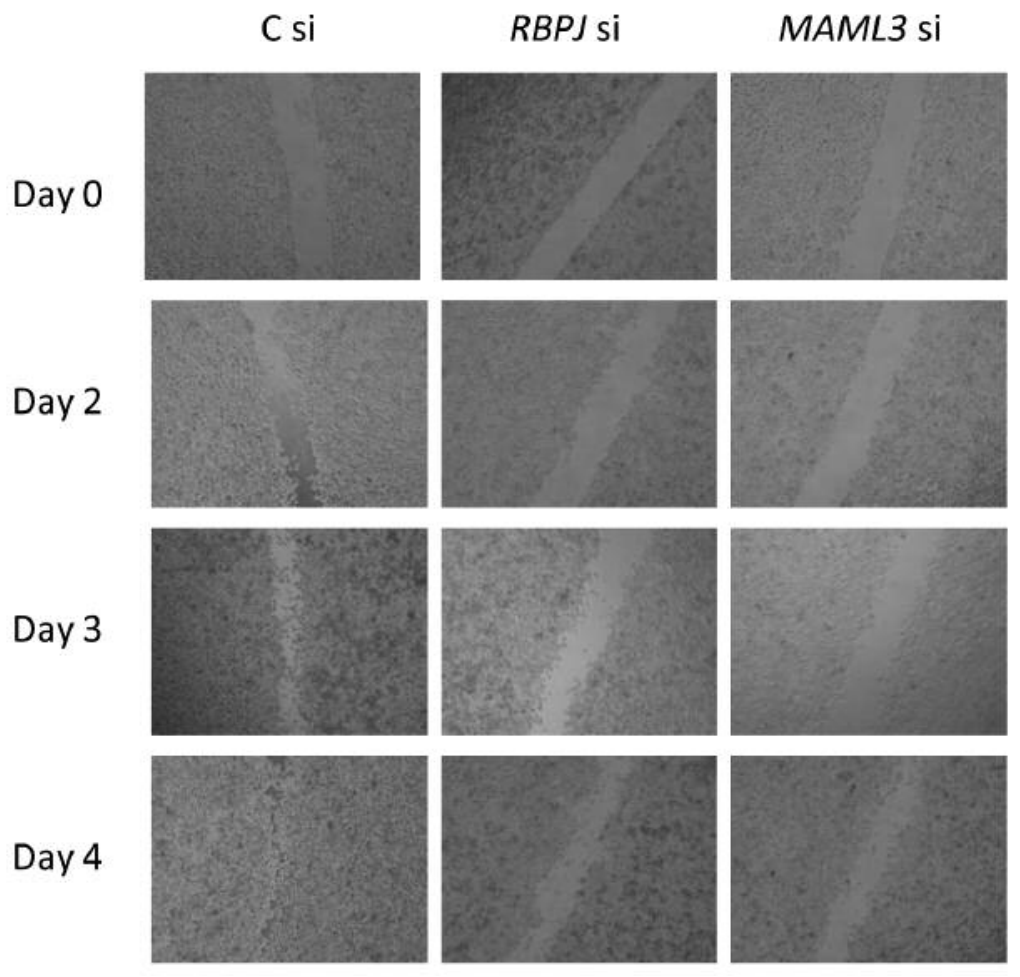

Figure 2. Inhibition of recombination signal binding protein for immunoglobulin-kappa-J region (RBPJ) and mastermind-like 3 (MAML3) delayed wound healing in small cell lung cancer cells. RBPJ siRNA (si)-transfected, MAML3 si-transfected and control si (C si)-transfected SBC-5 cells were used. Confluent cells were scratched by 24-G needle on day 0 then wound healing was observed at the indicated days. Representative images are shown $(\times 100)$.

\section{Results}

RBPJ and MAML3 contribute to proliferation, migration and invasiveness of SCLC cells. Firstly, we analyzed whether RBPJ and MAML3 are involved in biological functions in SCLC cells. Numbers of RBPJ siRNA-transfected cells and $M A M L 3$ siRNA-transfected cells after 3 days were significantly lower than those of control cells (Figure 1A). By inhibition of RBPJ and MAML3, numbers of migrated cells and invading cells significantly decreased compared with those of control cells (Figure 1B and C). In the woundhealing assay, wound closure by $R B P J$ siRNA transfected and $M A M L 3$ siRNA transfected SBC-5 cells was extremely delayed (Figure 2).

SMO and HES1 expressions in RBPJ siRNA transfected and MAML3 siRNA transfected cells were lower than those in controls, suggesting that signaling from RBPJ and MAML3 was through Hedgehog and NOTCH signaling pathways (Figure 3). MMP2 and MMP9 expression in RBPJ or MAML3 siRNA- transfected cells decreased compared to those of control cells (Figure 3). On the other hand, the expression of epithelial mesenchymal transition (EMT) markers; vimentin, E-cadherin and SNAI1 in RBPJ or
MAML3 siRNA- transfected cells showed no significant change compared to those of control cells (Figure 3). These results suggest that MMP2 and MMP9, but not changes in EMT may contribute to the mechanism of reduced invasiveness by inhibition of RBPJ and MAML3.

Inhibition of RBPJ and MAML3 reduced chemosensitivity of $S C L C$. Combinational use of chemotherapy drug and molecular targeting drug is an important strategy for refractory cancer. Therefore, chemosensitivity to combinational use of gemcitabine or CDDP with RBPJ/MAML3 inhibition was evaluated. The concentration of gemcitabine and CDDP was determined by previous experiment (15). Chemosensitivity to gemcitabine and CDDP decreased significantly when RBPJ and MAML3 were inhibited in SBC-5 cells (Figure 4).

\section{Discussion}

The purpose of this study was the development of a new effective molecular-targeting agent for refractory SCLC, which has few therapeutic strategies. As candidates, we selected RBPJ and MAML3 because we previously found that they were therapeutic targets for pancreatic cancer, 


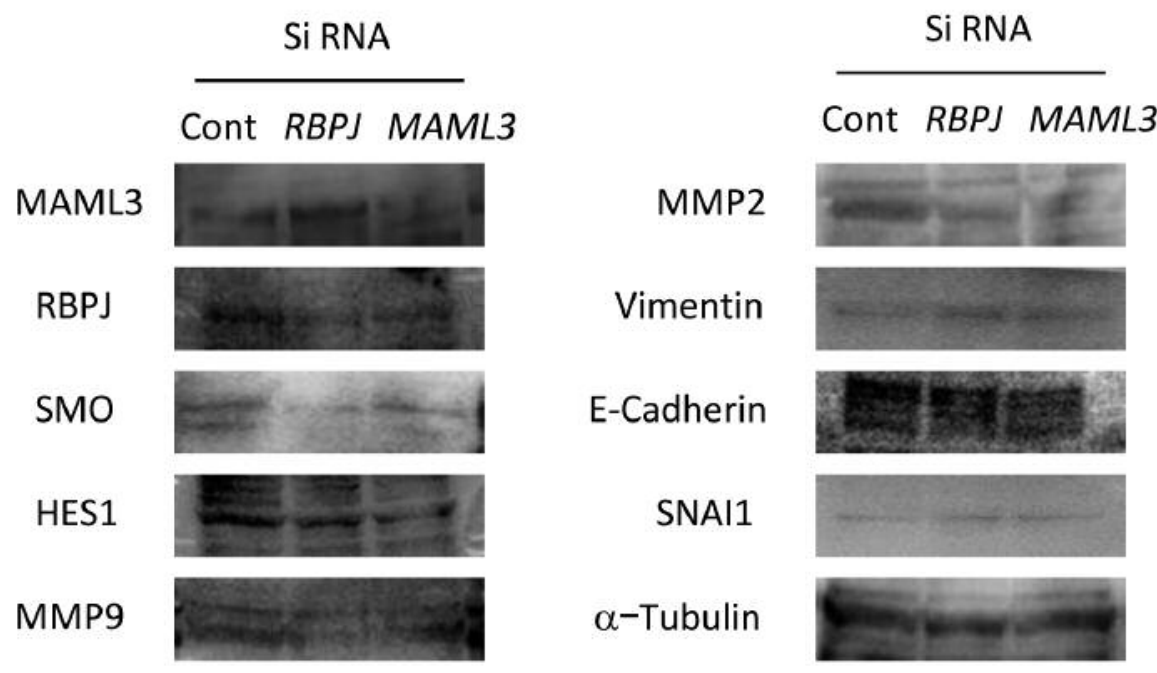

Figure 3. Inhibition of recombination signal binding protein for immunoglobulin-kappa-J region (RBPJ) and mastermind-like 3 (MAML3) reduced matrix metalloproteinase (MMP) expression in small cell lung cancer cells. RBPJ siRNA (si)-transfected, MAML3 si-transfected and control si (Cont)-transfected SBC-5 cells were assayed for protein expression of MAML3, RBPJ, Smoothened (SMO), HES1, and invasion-related molecules $M M P$, vimentin, E-cadherin, and SNAII by western blotting. $\alpha$-Tubulin was used as a loading control.
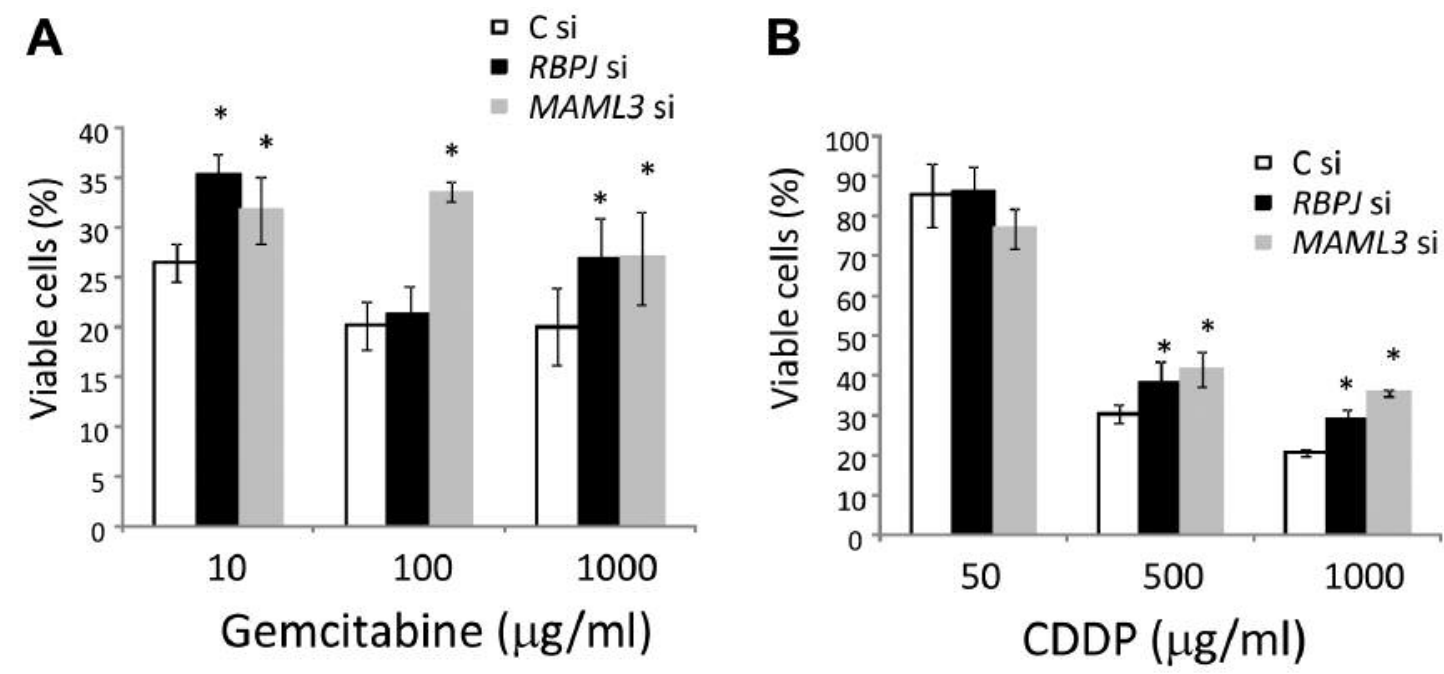

Figure 4. Inhibition of recombination signal binding protein for immunoglobulin-kappa-J region (RBPJ) and mastermind-like 3 (MAML3) reduced the sensitivity of small cell lung cancer (SCLC) cells to gemcitabine (A) and cis-diamminedichloroplatinum (CDDP) (B). RBPJ siRNA (si)-transfected, $M A M L 3$ si-transfected and control si (C si)-tranfected SBC-5 cells were treated for 2 days with gemcitabine or CDDP at the concentrations indicated. Viable SCLC cell numbers were then counted under light microscopy. Data are means \pm standard deviation. *Significantly different from control at $p<0.05$.

which is also a refractory type of neuroendocrine cancer (13). In the present study, siRNA was used to inhibit $R B P J$ and $M A M L 3$, because there were no specific inhibitors of RBPJ and MAML3 molecules. Inhibition of RBPJ and $M A M L 3$ was confirmed by western blotting, although inhibition was not complete (Figure 3).

As shown in Figure 3, signaling from RBPJ/MAML3 may be through Hedgehog and NOTCH signaling pathways in
SCLC similarly to pancreatic cancer (13). RBPJ and MAML3 are known activators of NOTCH signaling (12), while this is the first report that RBPJ/MAML3 may also serve as an activator of Hedgehog signaling in SCLC. Because both Hedgehog and Notch signaling are involved in morphogenesis, RBPJ/MAML3 inhibitors may be comprehensive morphogenesis signaling inhibitors against SCLC. 
In the mechanism of RBPJ/MAML3-induced invasion, MMPs, but not EMT are likely to be involved with the mechanism of invasiveness in SCLC as well as pancreatic cancer.

CDDP is the main agent for therapy of SCLC. Gemcitabine was also tested in this study, taking a new chemotherapy indication for SCLC into consideration, although gemcitabine is not approved for insurance coverage for SCLC in Japan (16). Although our results strongly imply that RBPJ and MAML3 could be new therapeutic targets for SCLC, one drawback is the resulting reduced sensitivity to CDDP by inhibition of RBPJ and MAML3. Gemcitabine also did not show an additive effect with inhibition of RBPJ and MAML3. Reduced chemosensitivity might be caused by the inhibition of proliferation due to suppression of RBPJ and MAML3. Therefore, although RBPJ and MAML3 might be therapeutically- targeted molecules for SCLC, caution is needed in their combinational use with other chemotherapy agents.

\section{Conflicts of Interest}

The Authors declare no financial or commercial conflict of interest in regard to this study.

\section{Acknowledgements}

The Authors thank Ms. Emi Onishi for skillful technical assistance. This study was supported by JSPS KAKENHI Grant Number $17 \mathrm{H} 04283$ and $18 \mathrm{~K} 08788$.

\section{References}

1 Noda K, Nishiwaki Y, Kawahara M, Negoro S, Sugiura T, Yokoyama A, Fukuoka M, Mori K, Watanabe K, Tamura T, Yamamoto $\mathrm{S}$ and Saijo N: Irinotecan plus cisplatin compared with etoposide plus cisplatin for extensive small-cell lung cancer. N Engl J Med 346: 85-91, 2002.

2 Faivre-Finn C, Snee M, Ashcroft L, Appel W, Barlesi F, Bhatnagar A, Bezjak A, Cardenal F, Fournel P, Harden S, Le Pechoux C, McMenemin R, Mohammed N, O'Brien M, Pantarotto J, Surmont V, Van Meerbeeck JP, Woll PJ, Lorigan P and Blackhall F: Concurrent once-daily versus twice-daily chemoradiotherapy in patients with limited-stage small-cell lung cancer (CONVERT): an open-label, phase 3, randomised, superiority trial. Lancet Oncol 18: 1116-1125, 2017.

3 Onishi $\mathrm{H}$ and Katano $\mathrm{M}$ : Hedgehog signaling pathway as a therapeutic target in various types of cancer. Cancer Sci 102: 1756-1760, 2011

4 Nagamatsu I, Onishi H, Matsushita S, Kubo M, Kai M, Imaizumi A, Nakano K, Hattori M, Oda Y, Tanaka $\mathrm{M}$ and Katano M: NOTCH4 is a potential therapeutic target for triplenegative breast cancer. Anticancer Res 34: 69-80, 2014.
5 Park KS, Martelotto LG, Peifer M, Sos ML, Karnezis AN, Mahjoub MR, Bernard K, Conklin JF, Szczepny A, Yuan J, Guo R, Ospina B, Falzon J, Bennett S, Brown TJ, Markovic A, Devereux WL, Ocasio CA, Chen JK, Stearns T, Thomas RK, Dorsch M, Buonamici S, Watkins DN, Peacock CD and Sage J: A crucial requirement for Hedgehog signaling in small cell lung cancer. Nat Med 17: 1504-1508, 2011.

6 Castellone MD, Laukkanen MO, Teramoto H, Bellelli R, Alì G, Fontanini G, Santoro M and Gutkind JS: Cross talk between the bombesin neuropeptide receptor and Sonic hedgehog pathways in small cell lung carcinoma. Oncogene 34: 1679-1687, 2015.

7 Szczepny A, Rogers S, Jayasekara WSN, Park K, McCloy RA, Cochrane CR, Ganju V, Cooper WA, Sage J, Peacock CD, Cain JE, Burgess A and Watkins DN: The role of canonical and noncanonical Hedgehog signaling in tumor progression in a mouse model of small cell lung cancer. Oncogene 36: 5544-5550, 2017.

8 Hassan WA, Yoshida R, Kudoh S, Hasegawa K, Niimori-Kita K and Ito T: Notch1 controls cell invasion and metastasis in small cell lung carcinoma cell lines. Lung Cancer 86: 304-310, 2014.

9 Hassan WA, Yoshida R, Kudoh S, Kameyama H, Hasegawa K, Niimori-Kita K and Ito T: NOTCH1 controls cell chemoresistance in small cell lung carcinoma cells. Thorac Cancer 7: 123-128, 2016.

10 Kikuchi H, Sakakibara-Konishi J, Furuta M, Yokouchi H, Nishihara H, Yamazaki S, Uramoto H, Tanaka F, Harada M, Akie K, Sugaya F, Fujita Y, Takamura K, Kojima T, Harada T, Higuchi M, Honjo O, Minami Y, Watanabe N, Oizumi S, Suzuki $\mathrm{H}$, Ishida $\mathrm{T}$, Dosaka-Akita $\mathrm{H}$, Isobe $\mathrm{H}$, Munakata $\mathrm{M}$ and Nishimura M: Expression of NOTCH1 and NUMB in small cell lung cancer. Oncotarget 8: 10348-10358, 2017.

11 Nam Y, Sliz P, Song L, Aster JC and Blacklow SC: Structual basis for cooperativity in recruitment of MAML coactivators to NOTCH transcription complexes. Cell 124: 973-983, 2006.

12 Tanigaki K, Han H, Yamamoto N, Tashiro K, Ikegawa M, Kuroda K, Suzuki A, Nakano T and Honjo T: NOTCH-RBPJ signaling is involved in cell fate determination of marginal zone B-cells. Nat Immunol 3: 443-450, 2002.

13 Onishi H, Yamasaki A, Kawamoto M, Imaizumi A and Katano M: Hypoxia but not normoxia promotes Smoothened transcription through up-regulation of RBPJ and Mastermindlike 3 in pancreatic cancer. Cancer Lett 371: 2419-2424, 2016.

14 Onishi H, Nakamura K, Nagai S, Yanai K, Yamasaki A, Kawamoto M, Imaizumi A and Morisaki T: Hedgehog inhibition up-regulates TRK expression to antagonize tumor suppression in small cell lung cancer cells. Anticancer Res 37: 4987-4992, 2017.

15 Onishi H, Morifuji Y, Kai M, Suyama K, Iwasaki H and Katano M: Hedgehog inhibitor decreases chemosensitivity to 5fluorouracil and gemcitabine under hypoxic conditions in pancreatic cancer. Cancer Sci 103: 1272-1279, 2012.

16 Japan Lung Cancer Society: Treatment for Lung Cancer (in Japanese), 2015.
Received June 8, 2018

Revised June 22, 2018

Accepted June 27, 2018 